\title{
OPTICAL, PROPERTIES OF FLYASH
}

Contract No. DE-AC22-87PC 79903

Quarterly Report for Period 1 January - 31 March 1992

Prepared for Pittsburgh Energy Technology Center

Principal Investigator Professor S. A. Self

April 1992

HIGH TEMPERATURE GASDYNAMICS LABORATORY Mechanical Engineering Department Stanford University 


\section{OPTICAL PROPERTIES OF FLYASH \\ Contract No. DE-AC22-87PC 79903 \\ Quarterly Report for Period 1 January - 31 March 1992 \\ Prepared for Pittsburgh Energy Technology Center \\ Principal Investigator Professor S. A. Self}

\section{EXECUTIVE SUMMARY}

The general aims of this research are to provide a fundamental scientific basis for the physical understanding and reliable calculation of radiative heat transfer in coal combustion systems, particularly as it is influenced by the presence of inorganic constituents deriving from the mineral matter in coal.

The work is organized under four tasks. Tasks I and II were initiated in October 1987; Tasks III and IV were funded from October 1988.

Task 1. Characterization of Flyash: Under this heading the chemical composition and size distribution of representative flyashes are being measured by appropriate microanalytical techniques to provide information required in Task 2 .

Task 2. Measurements of the Optical Constants of Slags: Under this heading measurements of the infrared optical constants (i.e., the complex refractive index $m=n-i k$ ) of synthetic slags are being made as a function of wavelength and temperature for controlled compositions. Particular attention will be given to the contribution of the $\mathrm{Fe}_{2} \mathrm{O}_{3}$ content and its valence state. The data is being reduced to yield formulae giving the complex refractive index over relevant ranges of wavelength and temperature, as a function of the relevant metal oxide constituents.

Task 3. Sample Calculations of the Radiant Properties of Flyash Dispersions: This component comprises various calculations to guide and evaluate the experimental work under the other three tasks.

Task 4. Measurement of the Radiant Properties of Flyash Dispersions: This benchscale experiment is planned to compare the measured radiant properties of a dispersion of well-characterized ash with computations based on data developed under the first two tasks.

In this eighteenth and final quarter, some further measurements have been completed under Task 4, as reported in the Quarterly Report, and summarized below. This essentially completes all the work planned under this contract.

The two Graduate Research Assistants involved in this work during the pas 4-1/2 years are in process of writing their Ph.D. dissertations which, when completed, will be issued as Technical Reports. A Final Report will be prepared in due course.

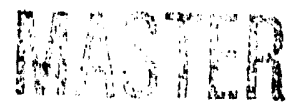




\section{Task 1}

The ashes being characterized are samples from power plants or pilot-scale combustors derived from combustion of six of the coals selected for study under the parallel PETC program on "Transformation of Inorganic Coal Constituents in Combustion Systems."

The principal features requiring characterization are particle size and composition distributions, including correlations between size and composition. Size distributions are being measured in house by Coulter counter. Size and composition distributions are being determined by automated SEM/microprobe analysis at UNDERC. Other in-house characterization work under way includes size classification by wet sieving combined with classification by density using flotation/sedimentation techniques, low temperature ashing for the char content and magnetic separation for the magnetite content resulting from combustion of pyrite.

Size distributions for a!l six ashes over the range $0.5-60 \mu \mathrm{m}$ have been completed using the Coulter multisizer with a technique employing two orifices to cover the whole range. A suitable technique for matching distributions using two orifice sizes was devised. The measured distribution was found to be very well represented by truncated log-normal distributions. Comparison with the size distribution from automated SEM shows some discrepancy.

Micrographs of the ash samples prepared by UNDERC for automated SEM/EDX sizecomposition analysis revealed many agglomerates, casting some doubt on the validity of the data. A freeze-drying technique for sample preparation has been developed at Stanford which gives very well dispersed samples. The automated SEM/EDX analysis has been repeated using such samples and the results have been analyzed using statistical computer codes developed at Stanford. Ternary diagrams of the composition distributions (in color) have been prepared using software developed at Stanford. Automated SEM/EDX analyses have been completed and analysis of the data made for all six ashes.

In addition, density classification results have been obtained for all six ashes and compared with results from SEM analysis ior composition, which, of course, determines the density. Size distributions have been determined for the density-separated fractions to obtain composition/size correlations for comparison with the SEM/EDX data. This task is now essentially completed. 


\section{Task 2}

Methods for determining the infrared optical properties of solid synthetic and natural slags at low temperatures were established in prior work at Stanford. In the present work the main effort has been devoted to the development of suitable apparatus and techniques for performing similar measurements on slags at temperatures to $2000 \mathrm{~K}$, when the slag is liquid. Basic experimental strategies were decided and apparatus designed to accomplish this task. Tests at high temperatures resulted in the first reliable measurements of the infrared absorption of liquid slag.

Two complementary techriques involving infrared optical measurements on liquid samples of synthetic slag maintained in an electric furnace have been developed. The first, for the wavelength range $1-5 \mu \mathrm{m}$ where the absorption index is low $\left(\mathrm{k} \leq 10^{-2}\right)$ employs a submerged platinum mirror to measure the absorption of thin films of slag by a double-pass technique. The second, applicable over the whole wavelength range $(1-12 \mu \mathrm{m})$, measures the surface reflectance of the liquid slag relative to that of a coid gold mirror in an external reference path.

The first technique was successfully used to obtain the first reliable measurements of the infrared absorption of liquid slag (at $2000 \mathrm{~K}$ ).

Efforts were then concentrated on developing and testing the second technique (for surface reflectivity measurements). It was determined that the quality of the data obtained is limited by the differing absorptions due to $\mathrm{CO}_{2}$ and $\mathrm{H}_{2} \mathrm{O}$ in the hot measurement path and the cold reference path. To eliminate this problem, the whole apparatus was enclosed in a chamber, purged with dry nitrogen.

With this modification, good measurements of the reflectivity of synthetic slag containing $5 \% \mathrm{Fe}$ at $1600^{\circ} \mathrm{C}$ were made over the whole wavelength range $1-12 \mu \mathrm{m}$. The data in the range 8-12 $\mu \mathrm{m}$ were reduced, using the Kramers-Kronig technique to give both the real and imaginary parts of the complex refractive index.

Possible sources of uncertainty in these measurements have been critically investigated and resolved. In particular, a problem due to contamination of a mirror by furnace gases has been identified and steps taken to eliminate it by redesign of the optical system. Reflectance measurements on liquid slags containing zero, 1\%, 5\%,10\% and $20 \%$ iron (as $\mathrm{Fe}_{2} \mathrm{O}_{3}$ ) have been completed. The results have been reduced, using Kramers-Kronig analysis to yield the real $(n)$ and imaginary $(k)$ parts of the complex refractive index.

A series of measurements were also made on liquid slags of varying $\mathrm{SiO}_{2}$ content. Work under this task is completed. 


\section{Task 3}

Programs have been written for Mie scattering calculations which are then convolved with input on the size and optical constants distributions for a particulate dispersion to yield the spectral scattering and absorption coefficients of the aerosol. Additionally, a program has been written to solve the radiation transfer problem for a homogeneous slab, utilizing the exact solution method of Case's normal modes. Input for the spectral scattering and absorption coefficients from the first program allows the spectral scattering, absorption and emission properties of the slab to be computed. These can then be integrated over wavelength to yield the total radiative heat transfer characteristics of the slab.

These programs have been used to determine the importance of certain features of typical ashes for radiation transfer. These include the sensitivity of the optical/radiative properties of a flyash dispersion to (i) composition-size correlation, especially with regard to the distribution of iron oxides with particle size, and (ii) the presence of bubbles in the glassy ash particles.

This computational capability was also used to evaluate the experimental conditions in the design of the apparatus for Task 4.

\section{Task 4}

Careful consideration was given to the feasibility of various basic approaches for implementing the goals of this task. After evaluating various experimental techniques, a basic approach was identified, which involves extinction measurements on flyash dispersed in suitable organic liquids. Measurements of the infrared transmission of three selected liquids were made which confirm their suitability for this purpose. $\mathrm{CaF} 2$ and $\mathrm{BaF} 2$ windows for an absorption cell were acquired and successful tests of a suitable cell design were made.

Careful consideration was given to the detailed optical design of a transmissometer for measurements on flyash dispersions in infra-red transmitting liquids, and a suitable design established.

Measurements of the extinction coefficient of dispersed samples of ashes from four of the coals have been made over the wavelength range $1-13 \mu \mathrm{m}$, and compared with calculations from the Mie code using input for the size and composition distributions from Task 1 .

Work under this task is now essentially complete. 


\subsection{INTRODUCTION}

This is the eighteenth quarterly report under DOE contract No. DE-AC22-87PC 79903 entitled "Optical Properties of Flyash." Tasks 1 and 2 of this program were funded from 15 September 1987. Tasks 3 and 4 were funded from 15 September 1988.

The general aims of this research are to provide a fundamental scientific basis for the physical understanding and reliable calculation of radiative heat transfer in coal combustion systems, particularly as it is influenced by the presence of inorganic constituents deriving from the mineral matter in coal. Some preliminary work in this area was carried out at Stanford earlier with NSF support. The present program greatly enlarges the scope of this work.

The complete, integrated program of theoretical and experimental work comprises four separate tasks.

Task 1. Characterization of Flyash

Task 2. Measurements of the Optical Constants of Slags

Task 3. Sample Calculations of the Radiant Properties of Flyash Dispersions.

Task 4. Measurements of the Radiative Properties of Flyash Dispersions.

In Task 1, the chemical composition and size distribution of representative flyashes are being measured by appropriate microanalytical techniques to provide information required in T'asks 2 and 3.

In Task 2, measurements of the infrared optical constants (i.e., the complex refractive index $m=n-i k$ ) of synthetic slags are being made as a function of wavelength and temperature for controlled compositions. Particular attention is being given to the contribution of $\mathrm{Fe}_{2} \mathrm{O}_{3}$ content and its valence state. The data will be reduced to yield formulae giving the complex refractive index over relevant ranges of wavelength and temperature, as a function of the relevant metal oxide constituents.

In Task 3, sample calculations are being made for typical ash loadings, size distributions and compositions for simple geometries, with two main purposes: first, to provide insight and physical understanding of the role of flyash in radiative heat transfer in combustion systems; second, to indicate the sensitivity of the results to the characteristics of the input data. Such calculations will also be used to determine appropriate conditions and to predict the expected measured radiative properties for the experiment of Task 4. 
The experiment of Task 4 is designed to critically test our ability to predict the measured spectral emittance and scattering coefficient of flyash dispersions under wellcontrolled laboratory conditions utilizing the optical property data developed in Task 2 . Particular attention will be paid to assessing the contribution of the char component in typical ashes. Any discrepancies between calculated and measured quantities revealed by these tests will be resolved by appropriate further studies.

A more detailed description of the scope of these tasks is given below. First, however, an outline is given of the rationale for the overall approach adopted in this program.

\subsection{Rationale of Overall Approach}

To account for the effects of flyash in radiative heat transfer calculations requires a knowledge of the contributions of the ash to the spectral absorption $\left(\mathrm{a}_{\lambda}\right)$ and scattering $\left(\sigma_{\lambda}\right)$ coefficients of the particulate dispersion, together with the phase function $\Phi_{\lambda}$ describing the anisotropy of the scattering. These quantities depend on the particulate loading as well as the distributions of the size and optical properties of the particles.

For a spherical particle of homogeneous, optically isotropic material, characterized by a complex refractive index $m \equiv(n-i k)$ Mie theory allows one to compute the spectral absorption $\left(Q_{\lambda, \mathrm{a}}\right)$ and scattering $\left(\mathrm{Q}_{\lambda, \mathrm{s}}\right)$ efficiencies of the particle, as well as the phase function $\phi_{\lambda}$. For randomly polarized radiation, these quantities are a function of the particle size parameter $x \equiv(\pi \mathrm{d} / \lambda)$, and the complex refractive index $\mathrm{m}(\mathrm{C}, \lambda, \mathrm{T})$, a function of composition, wavelength and temperature.

For a monodispersion of identical spherical particles, of specified loading (i.e. number density), the particulate's contribution to the optical properties $\left(a_{\lambda}, \sigma_{\lambda}\right.$ and $\left.\Phi_{\lambda}\right)$ of the medium are simply related to the spectral properties $\left(Q_{\lambda, a}, Q_{\lambda, s}, \phi_{\lambda}\right)$ of a single particle. It is also straightforward to compute the spectral optical properties of the medium for a polydispersion of spheres of identical composition, by convolving the results of Mie calculations for spheres of varying diameter (i.e. $x$ ) for fixed wavelength (and hence fixed $m$ ), with the particle size distribution (assumed given). In the case of a particulate material, like flyash, for which it is reasonable to assume that individual particles are of homogeneous composition but the composition varies from particle to particle, it is still possible to compute the spectral characteristics of the particulate dispersion by dividing the particles into an appropriate number of classes of varying composition (and hence $\mathrm{m}$ ), each having a specified size distribution, and summing over particle classes. 
In radiative heat transfer calculations, the contribution of the gas to the spectral absorption coefficient is added to that of the particles to obtain the combined optical properties of the medium on a spectral basis. These optical properties are then used as inputfor a radiation transfer code to calculate radiative fluxes, on a spectral basis, for a particular combustor geometry and boundary conditions. Finally, to obtain total heat transfer quantities such as the overall radiant heat flux, integrations over wavelength must be made.

The procedure, outlined above, represents the only logical approach to the computation of radiative heat transfer in flyash laden combustion gases. To implement this procedure requires, as input, a detailed characterization of the ash with respect to its size and (complex) refractive index distributions on a spectral basis.

Now, while techniques are available for determining the size distribution of powder samples, such as flyash, there are no practical means available for reliably determining the complex refractive index distribution of a complex material such as flyash either on a single particle basis, as a powder or as a dispersed aerosol. However, it is possible, using modern microanalytical techniques, specifically computer-automated SEM/EDX analysis, to determine the size and chemical composition of a heterogeneous powder on a particle by particle basis for a statistically large number of particles.

If the compositions of individual particles can be related to the complex refractive index of their material, then the characterization of a particular ash in terms of its size and composition distributions can lead to the necessary input for carrying out the calculations, outlined above, to compute radiation transfer in combustion systems containing that ash.

Thus the key requirement, necessary for the implementation of this approach, is data on the optical constants (i.e. the components $n, k$ of the complex refractive index) as a function of composition, wavelength and temperature covering the range of compositions found in representative ashes. Since, as noted above, and emphasized in texts on the optical properties of particulate matter, it is impractical to extract reliable data on the optical constants of material in particulate form, the only viable approach is to make measurements on homogeneous bulk samples for which well-established techniques are available.

The foregoing arguments provide the rationale for the present program. Characterization of representative flyashes concerning their size and composition distributions constitutes Task 1 , while measurements of the optical constants on bulk samples of synthetic slags as a function of relevant ranges of composition, wavelength and temperature constitute Task 2 . Task 3 is designed to provide computational capabilities to support the other tasks, while Task 4 is planned to provide an experimental test that the measured optical properties of a dispersion of flyash can indeed be computed reliably from a knowledge of the size and composition distributions of the ash. 


\subsection{Description of Tasks}

\section{TASK 1 - Characterization of Flyash}

Extensive prior analyses of flyash from a wide range of coals plus analyses of the mineral matter in raw coals, together with knowledge of the transformation processes occurring during combustion, lead to the following overall picture of the nature of flyash.

The particle size distribution is very broad with a volume (or mass) mean diameter on the order of $10 \mu \mathrm{m}$. 'Typically it is well represented by a log normal distribution with the $1 \%$ and $99 \%$ sizes in a cumulative plot by volume occurring at $\sim 1 \mu \mathrm{m}$ and $70 \mu \mathrm{m}$ respectively. Eviden $e$ of a distinct submicron fume due to homogeneous condensation of volatile mineral matter is sometimes found, but this fraction can be expected to contribute negligibly to radiation transfer.

With regard to chemical composition, several distinct classes of particle can be identified and plausibly related to their origin and formation mechanisms.

By far the preponderant class, usually representing on the order of $90 \%$ or more of the ash on a mass basis, consists of vitreous (amorphous) material composed primarily of $\mathrm{SiO}_{2}$, $\mathrm{Al}_{2} \mathrm{O}_{3}, \mathrm{CaO}$ and $\mathrm{MgO}$, usually in that order, but containing varying smaller percentages of other metal oxides, notably $\mathrm{Fe}_{2} \mathrm{O}_{3}$. It can appropriately be identified as particles of impure (calcium) aluminosilicate glass derived from the microscopic clay-like mineral inclusions in the coal matrix. As char burnout proceeds these inclusions melt and form liquid globules on the surface of the char (which they do not wet) and are then released into the gas.

These glassy particles tend to be quite spherical with smooth surfaces and of reasonably homogeneous composition as is to be expected from their formation as liquid droplets. As they cool after release from the char surface, they remain in the vitreous state because the cooling rate is much faster than the crystallization rate for the formation of specific phases. The fact that the bulk of most ashes consists of reasonably spherical, homogeneous and vitreous (and therefore optically isotropic) particles is a very fortunate fact, since they satisfy the assumptions of the Mie theory remarkably well.

Micrographs of optically polished sections of ash cast in epoxy resin, shows that these glassy particles sometimes contain a number of small bubbles of gas evolved from the char and trapped in the particles as they form on the char surface. More rarely, large, thin-walled cenospheres are observed which presumably are "glass-blown" when a liquid drop covers a pore in the char from which a relatively large volume of gas is evolved under pressure. Although such cenospheres are very prominent objects in micrographs, their number is usually too small to significantly affect radiation transfer. 
Auger spectroscopic studies of ash often show a thin surface layer composed of volatile metals and high in sulfur (as sulfates) and water. The presence of a thin layer of adsorbed water containing sulfate ions controls the electrical resistance of the surface which is very important in the performance of electrostatic precipitators. However, this surface layer, of different composition from the underlying particle, is too thin $(\leq 100 \AA)$ relative to wavelengths of interest to affect the optical properties of the particle.

Apart from this major class of glassy particles, several distinct minor classes of particle types can be identified, each comprising, at most, a few percent by mass of the flyash. One such class consists of incompletely burned char particles which are clearly identified in optical and SEM micrographs by the fact that they are black, of irregular shape and porous. The mass fraction of char depends on the particular coal and the combustor configuration and operating conditions. In modern combustors the mass fraction of unburned char is normally a few percent at most.

Another minor class consists of particles of adventitious incombustible mineral matter (e.g. quartz) which is contained in the pulverized coal feed. Such particles are usually large and of irregular shape, often showing rounded edges indicating partial melting.

A third minor class consists of magnetite $\left(\mathrm{Fe}_{3} \mathrm{O}_{4}\right)$ which derives from the combustion of pyrite $\left(\mathrm{FeS}_{2}\right)$ particles contained in the coal grind. These magnetite particles are black, generally spherical, magnetic and much denser than the glassy particles. The proportion of magnetite particles depends on the coal type, being largest in high sulfur coals, because the sulfur is mostly associated with pyrite. Recent work has shown that much of the pyrite in the coal grind can be removed by washing/sedimentation with a reduction in $\mathrm{SO}_{\mathrm{x}}$ emissions as high as $50 \%$ in some high sulfur coals.

The ashes selected for characterization are samples from power plants or pilot-scale combustors derived from the sarne seven coals selected for study under the parallel PETC program on "Transformation of Inorganic Coal Constituents in Combustion Systems" which comprise four bituminous, one sub-bituminous and two lignite coals.

A variety of techniques are being used in the characterization of these ashes. The principal method for determining size distributions employs a Coulter Multisizer which is capable of giving accurate, reliable results of high resolution over a wide dynamic range $\leq 1 \mu \mathrm{m}$ to $\geq 100 \mu \mathrm{m}$.

Automated, computer-controlled, combined SEM/EDX microanalysis will be the principal technique used to determine the distributions of composition and size for a large number $(\sim 1000)$ of particles for each ash. The size distributions will be compared with those obtained by the Coulter counter. 
Other techniques to be used include classification by density using liquids of varying density in a centrifuge, together with classification by size using a wet-sieving method. These techniques can yield density and size separated fractions for further examination by microanalytical techniques such as energy-dispersive X-ray spectroscopy. In addition, the magnetite particles may be separated by magnetic separation. The char content will be determined by low temperature ashing.

\section{TASK 2-Measurements of Optical Constants of Synthetic Slags}

This task is planned to provide the basic optical properties data in a comprehensive and conveniently usable form. The optical constants (i.e., the components of the complex refractive index $m=n-i k$ ) of samples of synthetic slags of controlled compositions will be measured using established techniques involving transmission and surface reflectance methods. The wavelength range will extend from the visible to $12 \mu \mathrm{m}$, and the temperature range will extend to $2000 \mathrm{~K}$.

In earlier work at Stanford, supported by NSF, extensive measurements of this type were made on polished wafers of synthetic slags at temperatures up to $1200 \mathrm{~K}$. A major component of this task will be to extend such measurements to higher temperatures $(\sim 2000 \mathrm{~K})$ where the slag is liquid. This requires the development of modified techniques which present a number of more or less severe technical challenges.

Initially, the optical constants of the basic calcium-aluminosilicate host glass will be determined for the composition range defined by Task 1 . Subsequently, by adding infraredactive mineral oxide constituents in controlled amounts, one at a time, the modifications to $\mathrm{m}(\lambda, \mathrm{T})$ produced by such constituents will be quantitatively determined. The particular constituents (and their range of mass fractions) to be examined will be determined by those disclosed by Task 1, taking account of knowledge of the optical activity at relevant wavelengths of such additions from the literature of glass technology. Specific constituents to be examined will include $\mathrm{Fe}_{2} \mathrm{O}_{3}$, taking especial account of its valence state $\left(\mathrm{Fe}^{2+} / \mathrm{Fe}^{3+}\right.$ ratio), and of $\mathrm{TiO}_{2}$. The contribution of the $\mathrm{OH}$ radical to the optical properties will be evaluated and quantified if significant.

The experimental data on $m(\lambda, T)$ as a function of composition, over the range relevant to coal ashes, will be reduced to generate simple correlation formulae. The latter will constitute the data base necessary to calculate the radiative properties of bulk slags and ash dispersions required for understanding and computing radiative transfer in coal combustion systems. 


\section{TASK 3 - Sample Calculations of the Radiant Properties of Flyash Dispersions}

This task is intended to provide computational capabilities to support the other tasks. It includes the following components.

(i) A Mie scattering code to calculate the absorption and scattering efficiencies and phase function of a single sphere of specified size parameter and complex refractive index. A modified Mie code will also allow such computations for hollow spheres.

(ii) A code to convolve the results from (i) over a specified size distribution and loading to compute the absorption and scattering coefficients and phase function of a homogeneous polydispersion.

(iii) A cole to sum the results of (ii) for a number of classes of particles of varying refractive indices and size distributions, i.e. for a heterogeneous polydispersion.

(iv) A radiation transfer code to calculate the absorption, scattering and emission characteristics of a homogenous, isothermal slab of dispersed ash on a spectral basis.

(v) A code to integrate the spectral results from (iv) over wavelength to yield the total radiative properties of the slab.

Calculations using these codes will be used to provide sensitivity analyses to guide the characterization work of Task 1, and to design and evaluate the results of Task 4.

\section{TASK 4-Measurement of the Radiative Properties of Flyash Dispersions}

The purpose of this laboratory scale experiment is to test our ability to predict the measured radiative properties of a dispersion of well-characterized flyash. As such it will provide a critical test of the effectiveness of the overall approach adopted in this program. 


\subsection{PROGRESS IN THE PAST QUARTER}

\section{1-2.3 Work under Tasks 1-3 is now completed.}

\subsection{Task 4: Measurement of Radiant Properties of Fly Ash Dispersions}

In this Task, a bench-scale experiment under controlled conditions in the laboratory is being perfnrmed to test the validity of the approach taken in the first three Tasks.

To be specific, the philosophy of our overall approach is the hypothesis that the optical/radiative properties of a particular fly ash dispersion can be predicted by appropriate codes (developed under Task 3) using input data on the size and composition distribution of the ash (determined using appropriate microanalytical techniques in Task 1), together with correlations for the spectral and temperature dependence of the complex refractive index as a function of ash composition (determined under Task 2 from measurements on synthetic slags). This fundamental approach has the advantage that the radiative properties of a particular ash (in a particular coal combustion environment) can be predicted purely by characterizing a sample of the ash using established microanalytical techniques, without having to resort to measurements of the optical/radiative properties of each ash, dispersed in a high temperature environment. Such measurements are extremely dificult (or impossible) to perform in a combustion environment, and it is this fact which led us to adopt the present philosophy.

To test the validity of this approach, Task 4 is intended to compare such predictions with measurements of the optical/radiative properties of a dispersion of a representative ash in a bench-style experiment under well-controlled conditions.

As described in past QPRs, a suitable bench-scale experiment was designed to satisfy the obiective of Task 4. A major design consideration was the conclusion that there is no known method for dispersing and adequately deagglomerating a sample of ash powder into a gas stream to reproduce the conditions of the ash aerosol before it was collected from the exhaust stream of the coal combustor. This conclusion was based on extensive experience of redispersing ash, and other fine, insulating powders into a gas stream for laboratory research in electrostatic precipitation. On the other hand, we found that it is possible to produce stable, well-deagglomerated dispersions of fly ash in liquids by using ultrasonic agitation. Moreover, by gentle stirring it is possible to maintain stable liquid dispersions 
at concentrations much higher than is possible with gaseous dispersions. This fact allows one to perform optical extinction and scattering experiments on a much smaller volume of dispersed ash (contained in an optical test cell) than is possible with a gaseous dispersion. This technique also permits the use of suitably high ash loadings to achieve measureable extinction.

On the basis of an experiment designed around the use of a liquid dispersion, attention was then focused on the choice of liquids and window material for a test cell to allow extinction and/or scattering experiments over the wavelength range of interest, say $\lambda=1-13 \mu \mathrm{m}$. While there is no liquid that is transparent over the whole range, it was established that by using three separate liquids, namely carbon tetrachloride $\left(\mathrm{CCl}_{4}\right)$, carbon disulfide $\left(\mathrm{CS}_{2}\right)$ and bromoform $\left(\mathrm{CHBr}_{3}\right)$, the full wavelength range could be covered. Moreover, it was also established that there is negligible chemical reaction between fly ash constituents and these liquids. For windows, barium fluoride $\left(\mathrm{BaF}_{2}\right)$ is suitable for the range $\lambda=1-13 \mu \mathrm{m}$.

In the QPR of February 1991, detailed descriptions of the experimental cell were presented. Mie scattering by particles suspended in a liquid medium was discussed. An alternate optical design of a transmissometer, suitable for our experimental conditions where the path length of the infra-red beam is comparable to the beam diameter, was described. Sensitivity calculations for extinction measurements for fly ash dispersed in $\mathrm{CCl}_{4}$ were presented in the QPR of May, 1991. The spectral refractive indices, $n(\lambda)$, of $\mathrm{CCl}_{4}, \mathrm{CS}_{2}$, and $\mathrm{CHBr}_{3}$, necessary for Mie calculations, were measured using the near-normal reflectance technique, and the results were reported in the QPR of July, 1991.

In the previous $Q P R$, results of transmittance measurements on suspensions of Upper Freeport ash in $\mathrm{CCl}_{4}$ were presented. It was explained why transmittance measurements were to be carried out on two density separated fractions of the Upper Freeport ashes. The fraction with density $<2.2 \mathrm{~g} / \mathrm{cc}$ consists entirely of cenospheres, while the fraction $>2.2 \mathrm{~g} / \mathrm{cc}$ can be expected to contain very few cenospheres. Results for the whole ash and the density separated fractions are presented here.

Two additional ashes, San Miguel and Eagle Butte, were also selected for transmittance measurements. The former ash consists almost entirely of cenospheres while the latter contains less than $5 \%$ cenospheres by mass. Hence, measurements on density separated ashes were not necessary for these two cases. 


\subsubsection{Transmittance Measurements on Fly Ash Suspensions}

The measured transmittance, $T$, is the ratio of the signal intensity transmitted through the ash suspension to that through the clean liquid. Hence, the extinction, $E$, by the ash dispersion is given by $(1-T)$. In the last QPR, transmittance measurements over the spectral range of 1-6 $\mu \mathrm{m}$ for Upper Freeport ash were presented. We discussed how the cenospheric fraction of the ash contributed to the spectral flattening of the transmittance curve. Last quarter, measurements of the ash dispersed in $\mathrm{CS}_{2}$ were carried for the whole ash as well as the density fractions $\rho_{<2.2}$ and $\rho>2.2$.

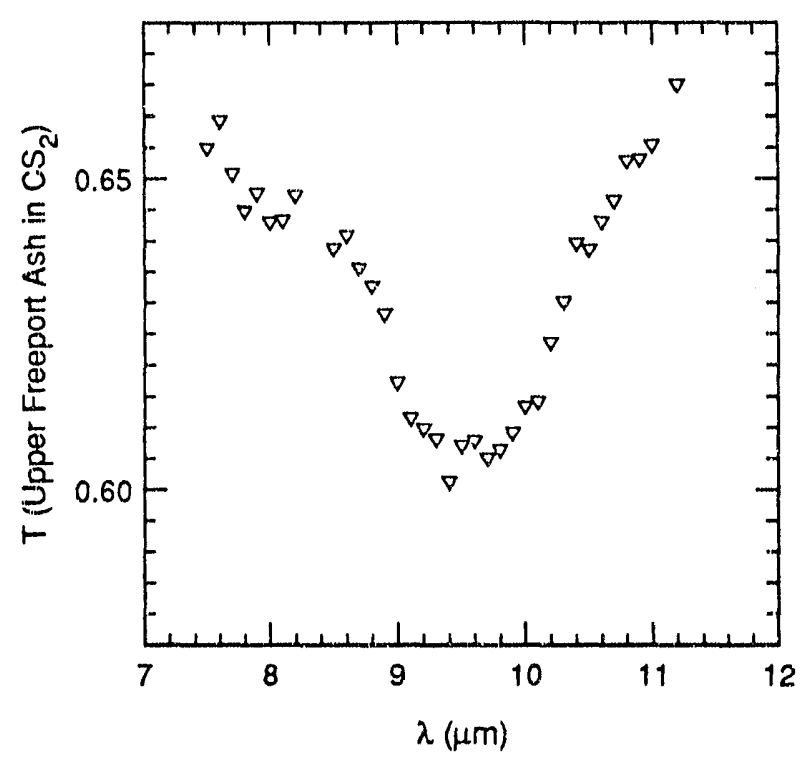

Figure 1: Transmittance Measurements on Cenospheric Upper Freeport Dispersed in $\mathrm{CS}_{2}$ (Density $=2.01 \mathrm{~g} / \mathrm{cc}$ ). Ash loading $=2 \times 10^{-4}$. Note expanded $\mathrm{y}$-axis.

The results are shown ir. Figures 1, 2, and 3. In all three cases, the broad absorption band is centered at $\approx 9.4 \mu \mathrm{m}$, and is attributed to the vibrational stretch of the bridging oxygen atom (Si-O-Si) (Simon, 1960). It is also interesting to note that there is no noticeable absorption due to the stretch vibrational mechanism of the non-bridging oxygen atom $\left(\mathrm{Si}-\mathrm{O}^{-}\right)$centered at $\approx 10.6 \mu \mathrm{m}$. Calculations for ash particles with the size distribution of Upper Freeport ash and composition of slag SA05 (Goodwin, 1986) are shown in Figure 4. Contributions to extinction by both the absorption and scattering components are shown. This range is the only part of the spectrum dominated by absorption. The scattering continues to decrease as $\lambda$ increases. Because the experiment is sensitive to $n(\lambda)$ and thus to the 


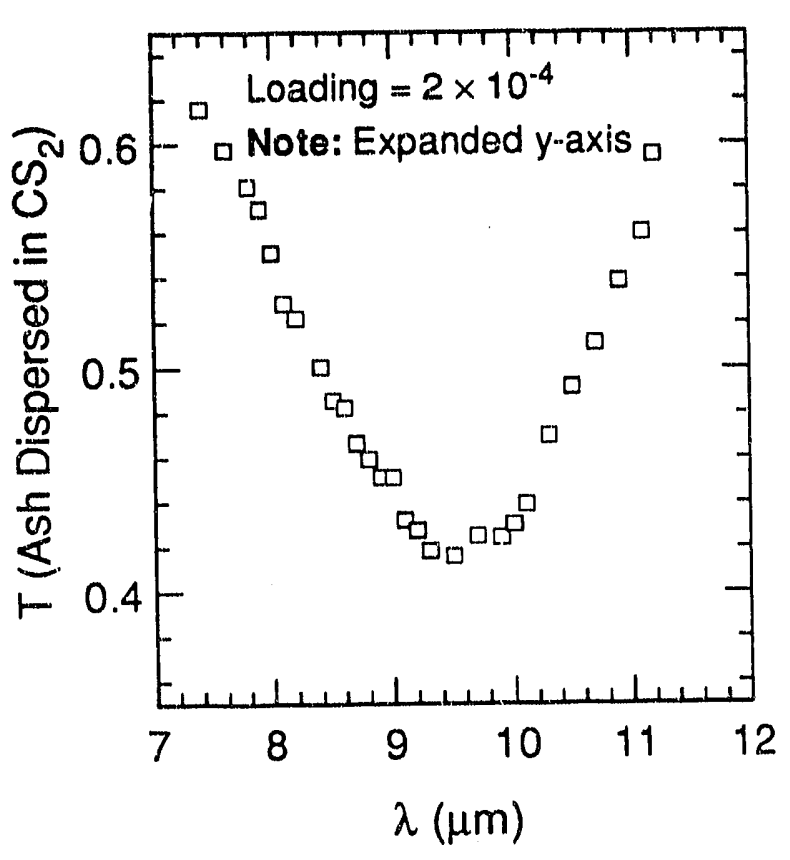

Figure 2: Upper Freeport Ash, $\rho>2.2 \mathrm{~g} / \mathrm{cc}$

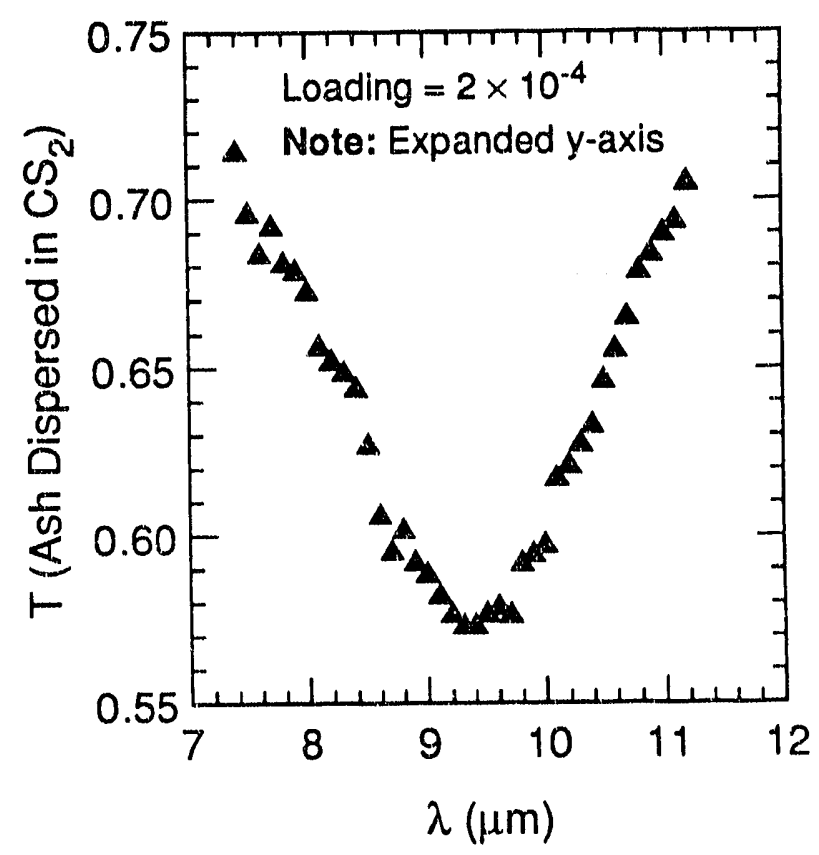

Figure 3: Upper Freeport Ash (Whole)

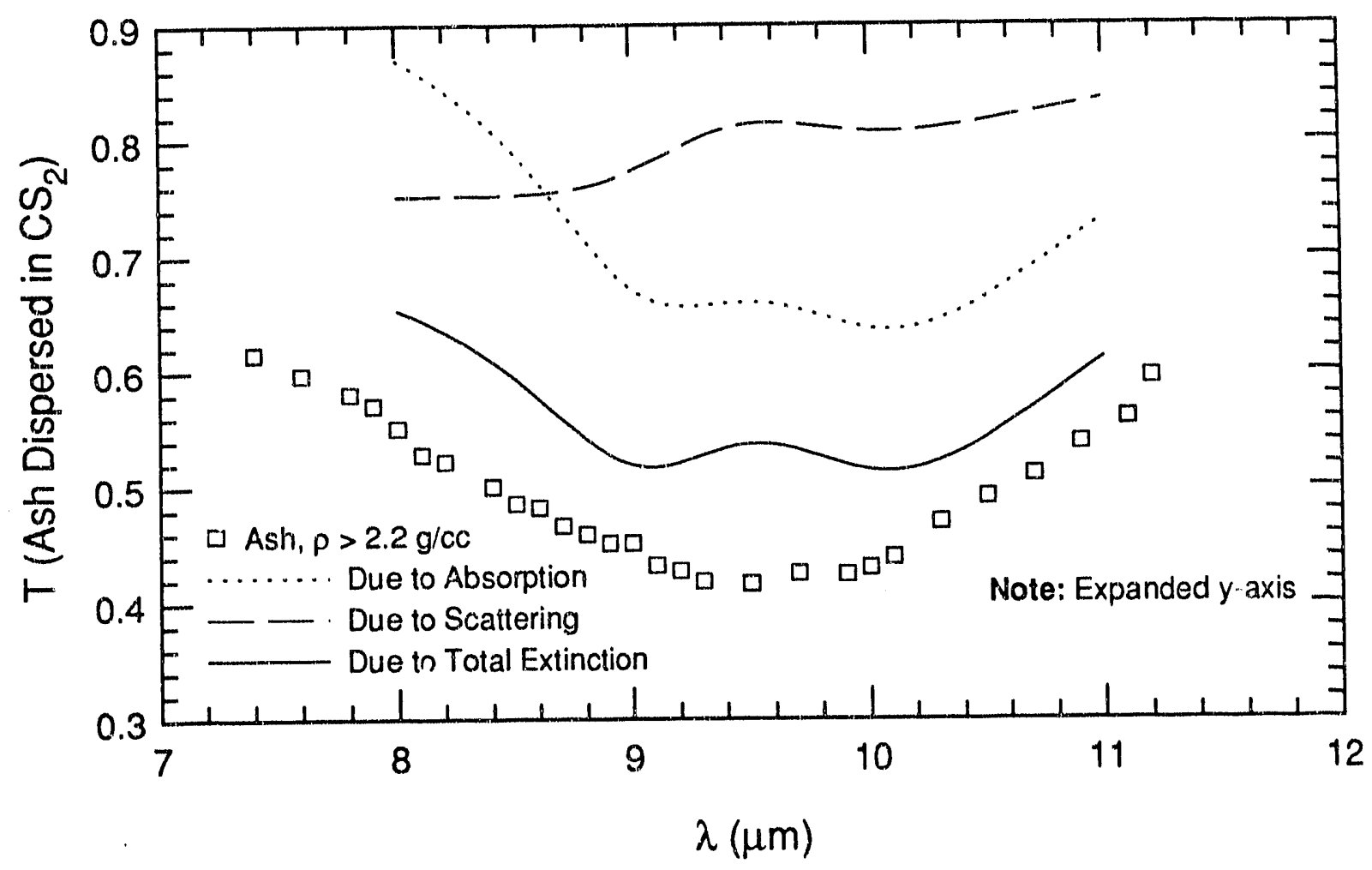

Figure 4: Upper Freeport Ash, $\rho>2.2 \mathrm{~g} / \mathrm{cc}$; Comparison with Calculations 
ash composition, the difference with the experimental data can be expected. The oscillator model, of course, assumes that both the vibration mechanisms explained above are active. From Figures 1 and 2, it is seen that the absorption by cenospheres is significantly lower than that by the solid ash particles. Because the cenospheres tend to be thin-walled, extinction is relatively small even at the strong restrahlen absorption band at $9.4 \mu \mathrm{m}$. Finally, the transmittance spectrum over the full range 1-13 $\mu \mathrm{m}$ is shown in Figure 5. The data for $\mathrm{CS}_{2}$ and $\mathrm{CHBr}_{3}$ were normalized with respect to the $\mathrm{CCl}_{4}$ data in order to obtain a continuous curve. This normalizing is essentially an adjustment of the ash loading.

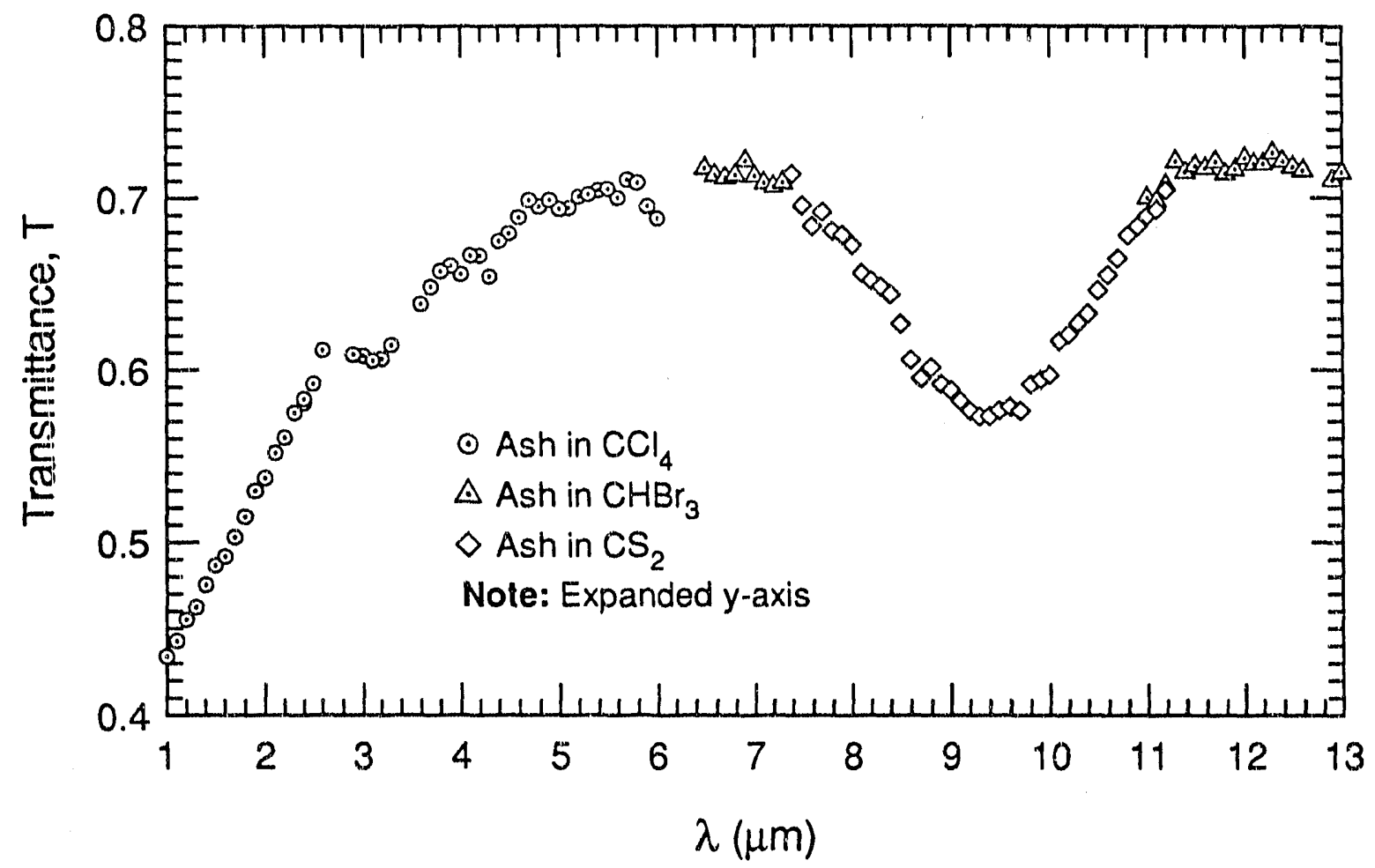

Figure 5: Transmittance Measurements on Upper Freeport over Wavelength Range 1-13 $\mu \mathrm{m}$. Note expanded y-axis.

The transmittance data for the San Miguel ash in $\mathrm{CCl}_{4}$ and $\mathrm{CS}_{2}$ are shown in Figure 6 and 7, respectively. This ash is almost entirely cenospheric (see previous QPR). As with the lighter density fraction of Upper Freeport ash, the extinction can be modeled approximately by air bubbles in the liquid. However, it is expected that Mie scattering calculations concentric, hollow spheres will give better results. Work in this regard is currently under way and the results will be included in the final report. The spectral extinction in the $7.5-11 \mu \mathrm{m}$ 


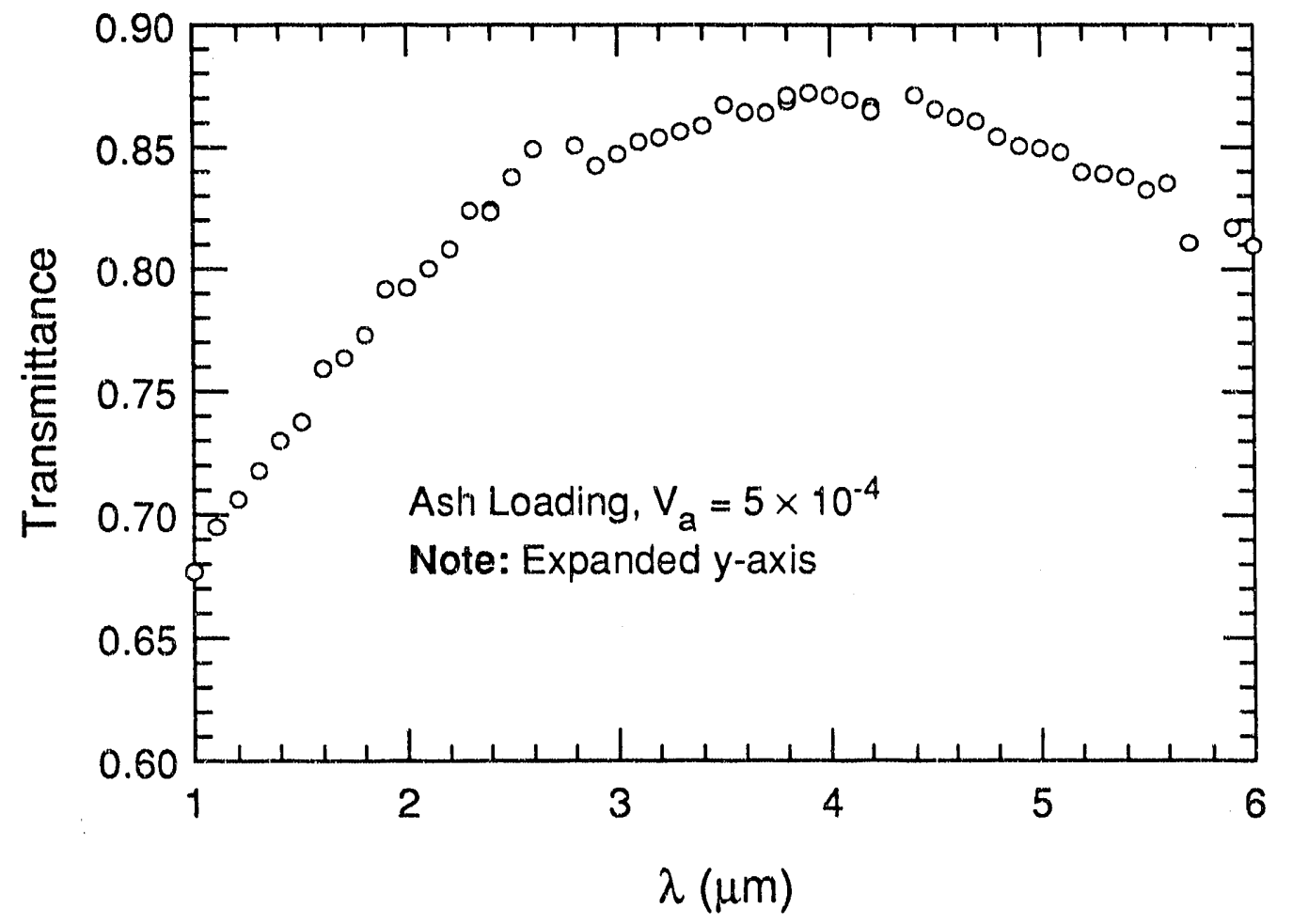

Figure 6: Transmittance of San Miguel Ash dispersed in $\mathrm{CCl}_{4}$

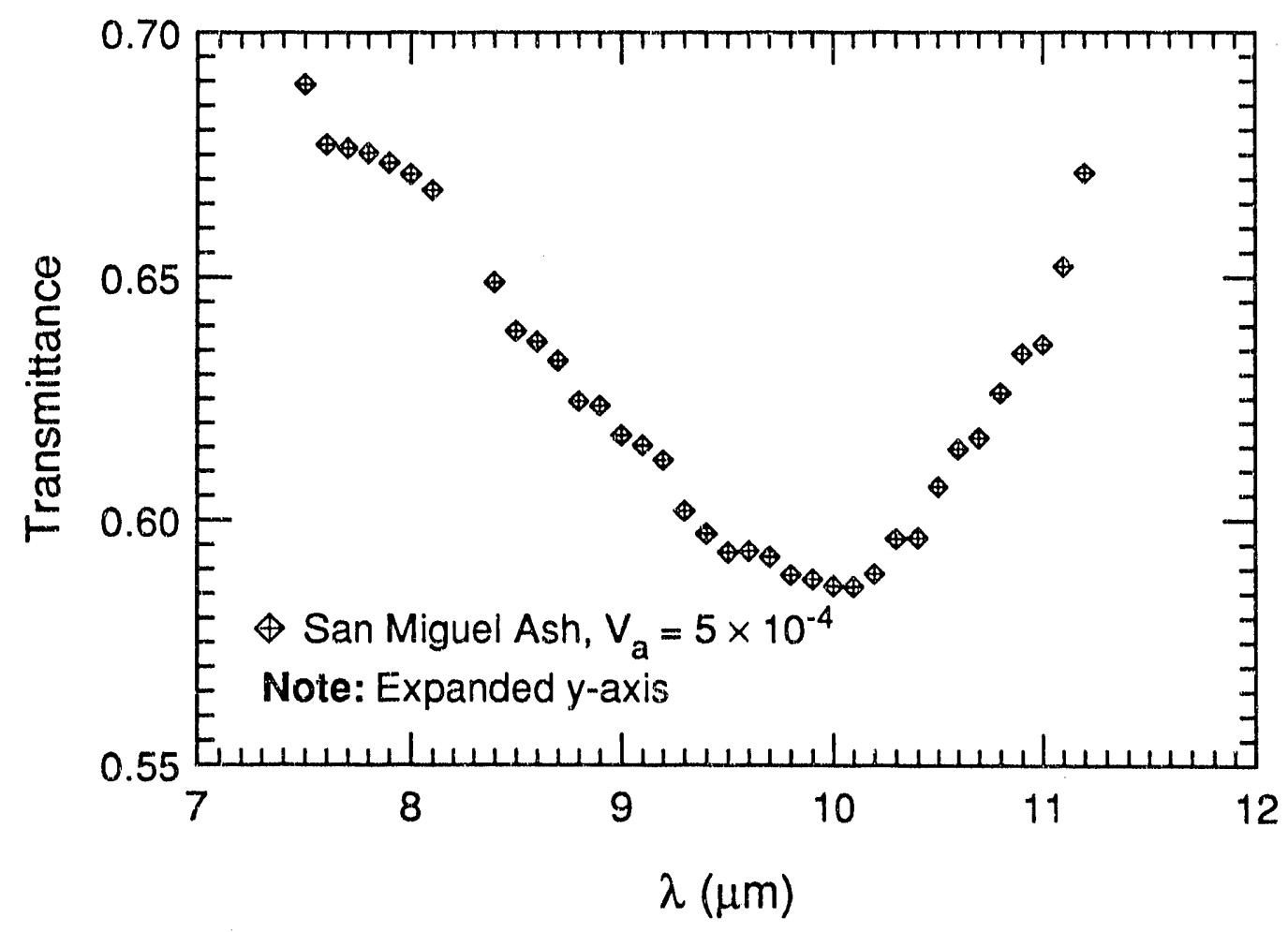

Figure 7: Transmittance of San Miguel Ash dispersed in $\mathrm{CS}_{2}$ 
range is similar to that of Upper Freeport, exhibiting the broad $\mathrm{Si}-\mathrm{O}-\mathrm{Si}$ absorption band centered at $\approx 9.8 \mu \mathrm{m}$.

Figures 8 and 9 on next page show the transmittance data for Eagle Butte ash. In contrast to the San Miguel ash, this ash contains very few cenospheres. As discussed in the previous QPR, the departure from the calculated transmittance in the wavelength range 3-6 $\mu \mathrm{m}$ is attributed to ash heterogeneity. The transmittance calculations assume that particles of all diameters have the same composition. The transmittance of the ash dispersion increases to almost $100 \%$ because the $n_{r e l}$ of all particles reaches a value of unity at the same wavelength. However, as we learnt from the CCSEM data, chemical composition varies significantly from particle to particle. Hence, $n_{\text {rel }}$ reaches unity at different wavelengths for different particles, which results in a flattening of the spectral transmittance peak. Calculations are currently underway to incorporate this variation of $n_{\text {rel }}$ in our model. Results will be discussed in the final report.

Unlike the previous two ashes, Eagle Butte ash shows at least two prominent absorption bands attributable to silica (Figure 9). While we have not yet identified the relatively narrow band centered at $\approx 8.7 \mu \mathrm{m}$, absorption attributable to the two mechanisms described above can clearly be seen. We made additional transmittance measurements on nominally monodisperse glass spheres of diameter $8 \mu \mathrm{m}$, and of the following average composition specified by the manufacturer: $\mathrm{SiO}_{2}=73 \%, \mathrm{Na} 2 \mathrm{O}=13 \%, \mathrm{CaO}=10 \%, \mathrm{MgO}=3.3 \%, \mathrm{Al}_{2} \mathrm{O}_{3}=0.4 \%$, $\mathrm{K}_{2} \mathrm{O}=0.1 \%, \mathrm{Fe}_{2} \mathrm{O}_{3}<0.2 \%$. The transmittance data in Figure 10 is similar to the results obtained with the Eagle Butte ash. Both indicate significant absorption by $\mathrm{SiO}^{-}$.

Engelhardt et al (1985) studied the structures of about fifty glasses in the $\mathrm{CaO}-\mathrm{Al}_{2} \mathrm{O}_{3}-$ $\mathrm{SiO}_{2}$ system. In glasses of composition similar to Eagle Butte (molar proportions of $\mathrm{CaO}$ $: \mathrm{Al}_{2} \mathrm{O}_{3}: \mathrm{SiO}_{2}=48: 14: 38$ ), three of the four oxygen atoms in $\mathrm{SiO}_{4}$ were bridging ones, with either two or all three $\mathrm{O}$ atom connecting the Si atom under consideration to $\mathrm{Al}$ atoms. As the molar concentration of $\mathrm{Ca}$ decreases to $<15 \%$, all four $\mathrm{O}$ atoms become bridging atoms, with either one or two connecting the Si atom to $\mathrm{Al}$ atoms. However, it is also known that the presence of significant amounts of network modifiers (e.g., $>5 \% \mathrm{Na}_{2} \mathrm{O}$, molar) in this glass forming system of $\mathrm{SiO}_{2}-\mathrm{Al}_{2} \mathrm{O}_{3}$ leads to the creation of non-bridging oxygen, $\mathrm{SiO}^{-}$ (Seifert et al 1982).

While comprehensive studies on the optical propeties of calcia rich aluminosilicate glasses are not available, the above observations appear to qualitatively explain the transmittance 


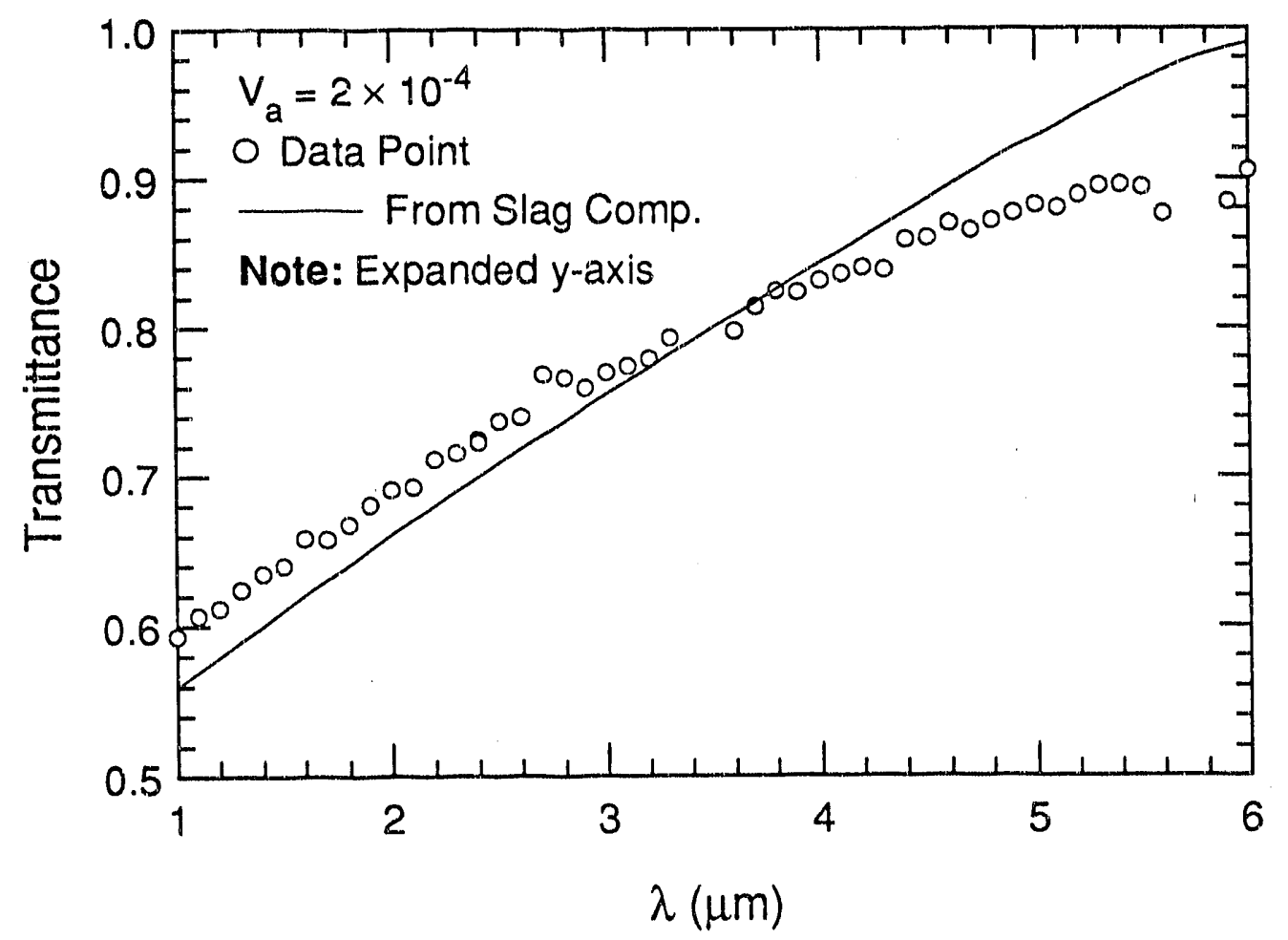

Figure 8: Transmittance of Eagle Butte Ash dispersed in $\mathrm{CCl}_{4}$

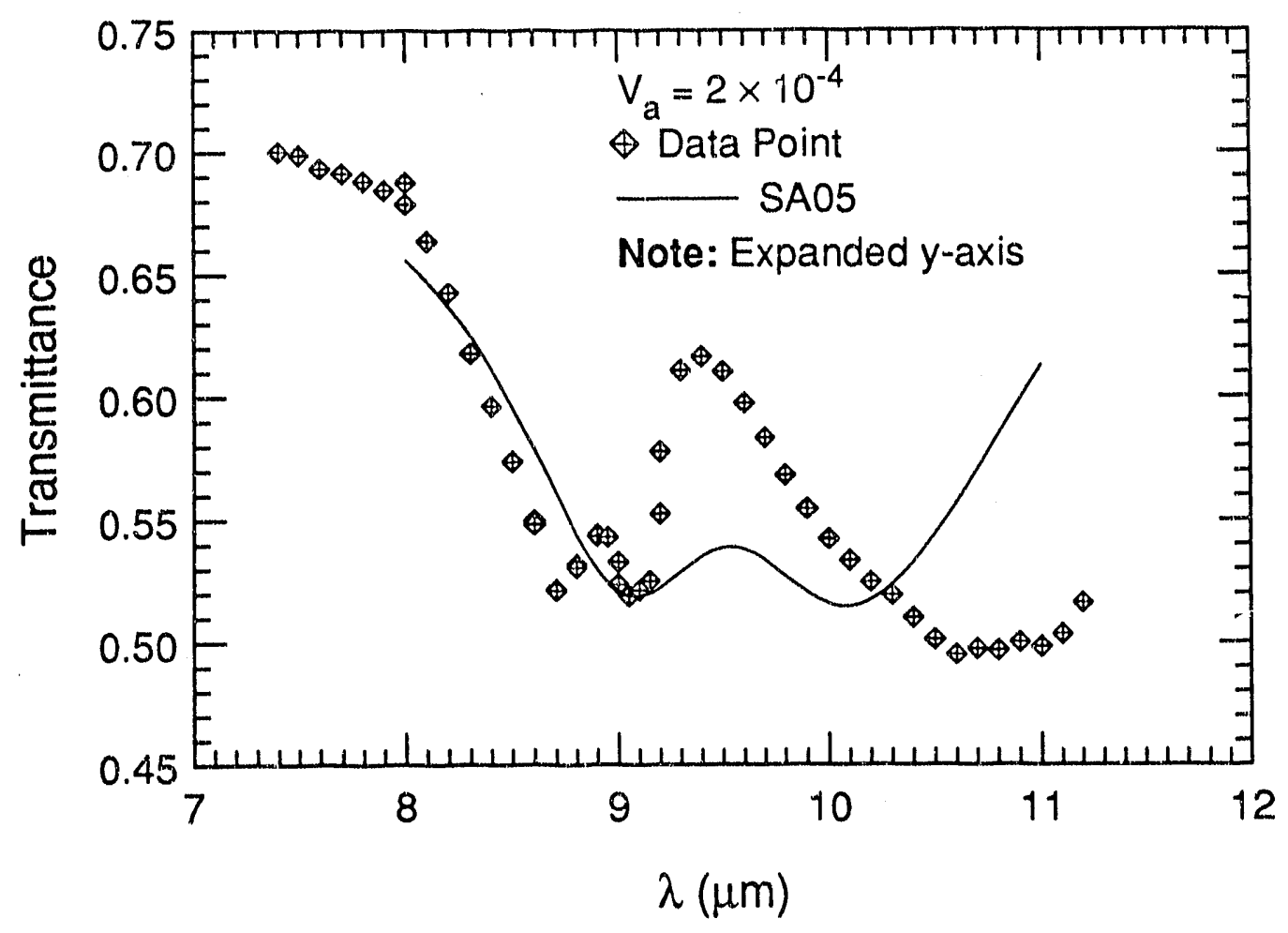

Figure 9: Transmittance of Eagle Butte Ash dispersed in $\mathrm{CS}_{2}$ 
data. Additionally, it should be noted that fly ash contains other impurities like Fe and $\mathrm{Mg}$ that modify the $\mathrm{SiO}_{4}$ network in extremely complex ways. We are continuing to search for literature on glass structure that describes the proportions of binding to non-binding oxygen atoms in aluminosilicate glasses containing other network modifiers.

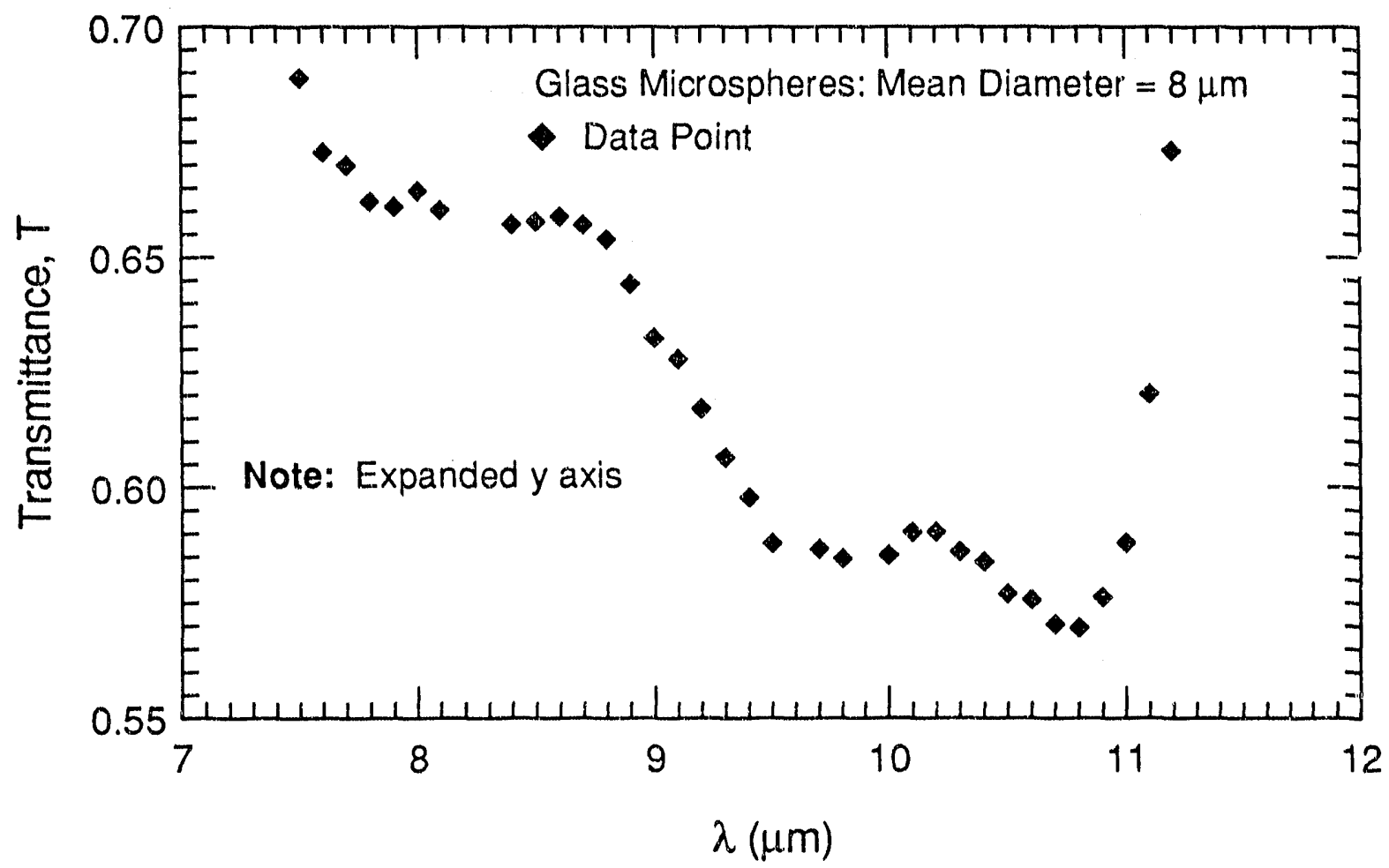

Figure 10: Transmittance Measurements on Glass Microspheres (Density=2.42 $\mathrm{g} / \mathrm{cc}$ ) dispersed in $\mathrm{CS}_{2}$. Loading $=2 \times 10^{-4}$. Note expanded $\mathrm{y}$-axis.

Scheduled Activities: Transmittance measurements are now almost complete. During the current quarter, some of the experiments will be re-run to obtain precision estimates. Further calculations are currently being performed to compare experimental data with predictions from Task 1 and 3 .

\section{References}

[1] Engelhardt, G., Nofz, M., Forkel, K, Wihsmann, F. G., Magi, M., Samoson, A., Lippmaa, E., 'Structural Studies Calcium Aluminosilicate Glasses by High Resolution 
Solid State ${ }^{29} \mathrm{Si}$ and ${ }^{27} \mathrm{Al}$ Magic Angle Spinning NMR', Physics and Chemistry of Glasses, Vol. 26, No. 5, pp. 157-165 (1985).

[2] Goodwin, D. G. Infrared Optical Constants of Coal Slags, Report T-255, High Temperature Gas Dynemics Laboratory, Stanford University (1986).

[3] Seifert F., Mysen, M. O., and Virgo, D., 'Three-Dimensional Network Structure of Quenched Melts (Glass) in the Systems $\mathrm{SiO}_{2}-\mathrm{NaAlO}_{2}, \mathrm{SiO}_{2}-\mathrm{CaAl}_{2} \mathrm{O}_{4}$ and $\mathrm{SiO}_{2}-$ $\mathrm{MgAl}_{2} \mathrm{O}_{4}$ ', American Mineralogist, Vol. 67, pp. 696-717 (1982).

[4] Simon, I., 'Infra-Red Studies of Glass', in Modern Aspects of the Vitreous State, ed. Mackenzie, J. D., Butterworth, Washington (1960). 


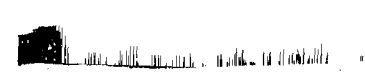

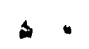
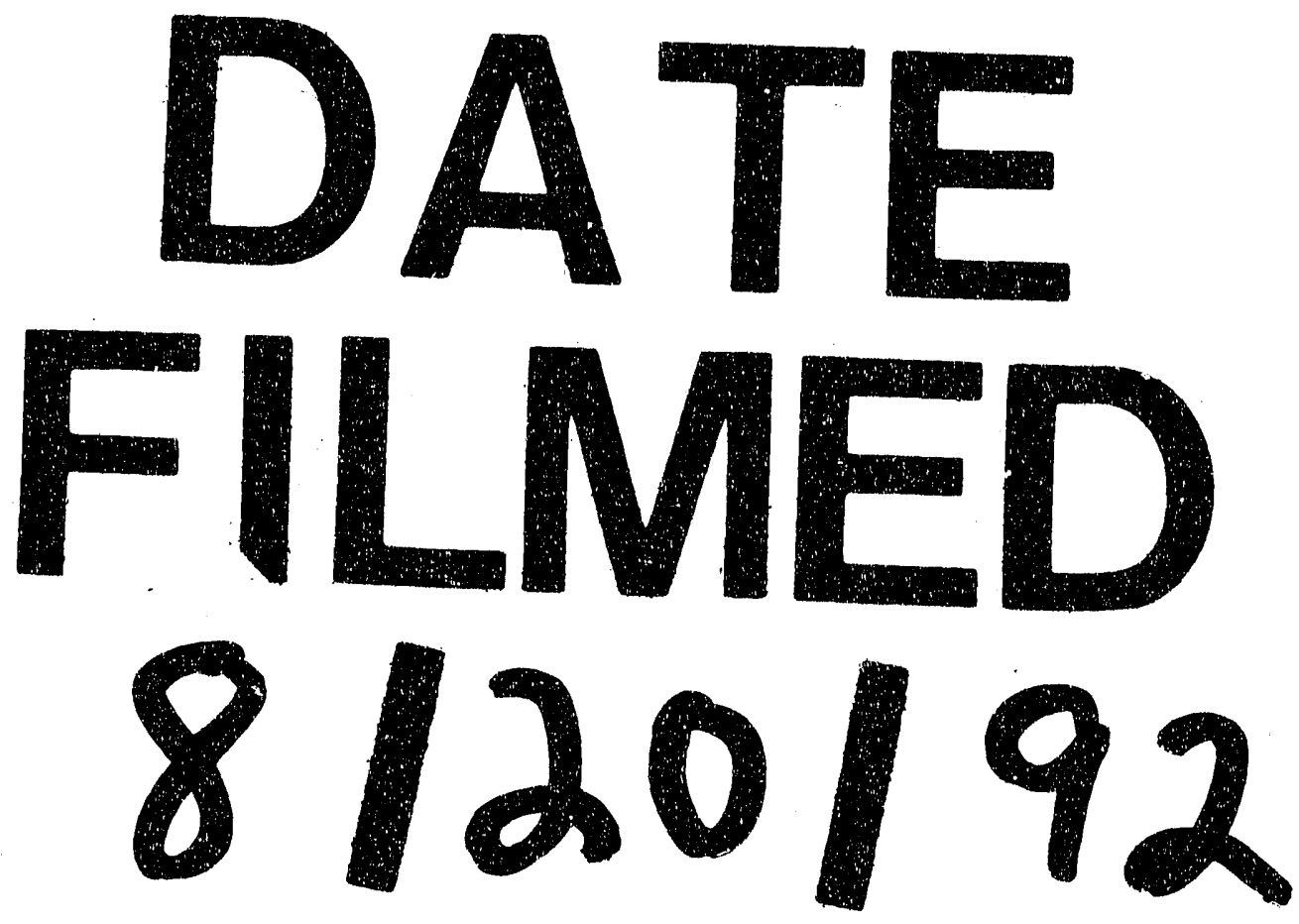

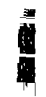


